\title{
Diabetes improvement and bariatric surgery-review of laparoscopic Roux-en-Y gastric bypass vs. laparoscopic vertical sleeve gastrectomy
}

\author{
Jeffrey Gu ${ }^{1}$, Ashley Vergis ${ }^{2}$ \\ ${ }^{1}$ Community Health and Epidemiology, Department of General Surgery, University of Saskatchewan, Saskatoon, Canada; ${ }^{2}$ Section of General \\ Surgery, Department of Surgery, Rady College of Medicine, Max Rady Faculty of Health Sciences, University of Manitoba, Winnipeg, Canada \\ Contributions: (I) Conception and design: All authors; (II) Administrative support: All authors; (III) Provision of study materials or patients: All \\ authors; (IV) Collection and assembly of data: All authors; (V) Data analysis and interpretation: All authors; (VI) Manuscript writing: All authors; (VII) \\ Final approval of manuscript: All authors. \\ Correspondence to: Jeffrey Gu, MD, PhD, FRCSC. Community Health and Epidemiology, Department of General Surgery, University of \\ Saskatchewan, Box 7, Health Science Building, 107 Wiggins Road, Saskatoon, SKS7N 5E5, Canada. Email: jeg998@mail.usask.ca.
}

\begin{abstract}
Obesity and type 2 diabetes mellitus (T2DM) are globally escalating major health care issues. For both obesity and T2DM management, it has been well established that bariatric surgery is superior to lifestyle and medical management alone. Over the past two decades, the introduction of laparoscopic vertical sleeve gastrectomy (LVSG) has seen a marked rise in usage, and combined with laparoscopic Roux-en-Y gastric bypass (LRYGB), these two procedures represent more than $80 \%$ of all bariatric surgeries globally. However, the differences in effectiveness between these two procedures have been less clearly defined. This article will serve as a focused review of the literature comparing LRYGB and LVSG for T2DM management. Based on our review, we believe that both procedures are very effective at improving T2DM care, especially compared with conventional medical management. However, there may be a modest benefit to be had by using LRYGB over LVSG.
\end{abstract}

Keywords: Bariatric surgery; Roux-et-Y gastric bypass (RYGB); sleeve gastrectomy; type 2 diabetes mellitus (T2DM)

Submitted Nov 12, 2019. Accepted for publication Jan 06, 2020.

doi: $10.21037 /$ atm.2020.01.47

View this article at: http://dx.doi.org/10.21037/atm.2020.01.47

\section{Introduction}

Obesity and type 2 diabetes mellitus (T2DM) are globally escalating major health care issues that are associated with significant morbidity, mortality, and healthcare expenditures (1-3). For both obesity and T2DM management, it has been well established that bariatric surgery is superior to lifestyle and medical management alone (4-7). Traditionally, laparoscopic adjustable gastric band (LAGB), vertical banded gastroplasty (VBG), and Roux-en-Y gastric bypass (RYGB) have been the most commonly performed procedures. However, over the past two decades, both LAGB and VBG have been all but abandoned as the introduction of laparoscopic vertical sleeve gastrectomy (LVSG) has seen a marked rise in usage, even overtaking RYGB in many regions (8). Globally, laparoscopic RYGB (LRYGB) and LVSG currently represent more than $80 \%$ of all bariatric surgeries (9). In view of the increasing use of LVSG, this article will serve as a focused review of the literature comparing LRYGB and LVSG for T2DM management.

\section{Bariatric surgery versus conventional medical management for T2DM}

The literature evaluating bariatric surgery and conventional medical management for T2DM is robust. This includes a recent systematic review and meta-analysis by Ribaric 
et al. involving 6,131 patients from 16 randomized control trials (RCTs) and observational studies (6). The majority of bariatric procedures performed were LRYGB, but LAGB, LVSG, and biliopancreatic diversion (BPD) were also included. With a mean follow-up time of 17.3 months, the T2DM remission rates for surgery versus conventional medical management were $63.5 \%$ and $15.6 \%(\mathrm{P}<0.01)$ respectively. The researchers reported a pooled odds ratio (OR) for T2DM remission of 9.8 (95\% CI: 6.1-15.9) and an inverse pooled OR of 15.8 (95\% CI: 7.9-31.4), clearly indicating superior remission with bariatric surgery. Other current systematic reviews also reported similar remission rates with bariatric surgery: Gill et al. reported a rate of $66 \%$ with LVSG (10), and Buchwald et al. reported a rate of $76.8 \%$ with mixed bariatric surgeries (4).

The long-term durability of bariatric surgery on T2DM treatment is not as well studied. The landmark Swedish Obese Subjects (SOS) matched intervention trial likely provides the best idea of long-term outcomes (11). Bariatric surgery in this study included LAGB, VBG, or gastric bypass. At 2 years, T2DM remission rate in post-bariatric surgery patients was $72.0 \%(\mathrm{n}=342)$, compared with $21.0 \%$ $(\mathrm{n}=248)$ in the medical therapy group (OR: 8.42, $\mathrm{P}<0.01)$. At 10 years, the remission rate in the post-bariatric surgery group dropped to $37.0 \%(\mathrm{n}=118)$, compared with $12.0 \%$ $(\mathrm{n}=84)$ in the medical therapy group $(\mathrm{OR}: 3.45, \mathrm{P}<0.01)(11)$. Interestingly, this large trial also reported a T2DM risk reduction effect of surgery, compared with usual care of $96 \%, 84 \%$, and $78 \%$ after 2,10 , and 15 years, respectively $(11,12)$. Thus, in contrast to declining remission effect on T2DM with time, the strong prevention effect was only moderately reduced at prolonged follow-up.

Five-year results from the STAMPEDE trial corroborate the findings from the SOS study (13). This RCT compared intensive medical therapy with bariatric surgery (LVSG or LRYGB) in moderately obese individuals (BMI: 27 to 43) who had T2DM. At 5 years, among 134 individuals, diabetes remission was seen in 2 of 38 patients (5\%) who received intensive medical therapy alone, compared with 14 of 49 patients (29\%) who underwent LRYGB ( $\mathrm{P}=0.03)$, and 11 of 47 patients (23\%) who underwent LVSG $(\mathrm{P}=0.07)$. These differing remission rates between studies might arise from differences in end point definitions: The SOS used a fasting blood glucose of $<110 \mathrm{mg} / \mathrm{dL}$ and off diabetes medication, whereas the STAMPEDE trial used a hemoglobin A1c (HbA1c) level of $\leq 6.0 \%$ with or without the use of medications.

\section{LRYGB vs. LVSG for T2DM management}

The literature evaluating LRYGB vs. LVSG for T2DM management is sparse in comparison with that for bariatric surgery $v s$. conventional medical treatment. We identified only five systematic reviews that have been published on this topic (14-18). What's more, the early reviews suffer significant limitations owing to the paucity of published literature. Although we can draw on a number of welldesigned RCT's evaluating weight loss with LRYGB $v s$. LVSG that report secondary outcomes in T2DM management, specific studies designed to evaluate diabetes improvement with LRYGB $v s$. LVSG are sparse. Likewise, long-term outcomes comparing the two procedures are scant.

\section{Systematic reviews}

The most robust systematic review was published in 2018 and was conducted by Hayoz et al. (17). This review excluded any observational studies and included RCTs only. Sixteen RCTs, totaling 1,132 patients, of obese patients with or without DM were included in the review. Many of these studies reported on the same cohort of patients (follow-up publications), with only the most recent data included. The primary outcomes for T2DM were improvements in fasting blood glucose levels, insulin resistance, and HbA1c. Hayoz et al. conducted primary analysis of the entire cohort and subanalysis of included studies that investigated only obese patients who had T2DM.

Fasting blood glucose demonstrated no difference between groups at 12 months but a significant difference favoring LRYGB at 24 months [mean difference (MD): $-16.92 \mathrm{mg} / \mathrm{dL}, 95 \% \mathrm{CI}:-21.67$ to $-12.18 ; \mathrm{P}<0.01]$ and 36 months (MD: $-5.97 \mathrm{mg} / \mathrm{dL}, 95 \%$ CI: -9.32 to -2.62 ; $\mathrm{P}<0.01$ ). Only 3 studies reported on fasting insulin levels, and they had similar values between groups at 12 -month follow-up. For HbA1c, a significant difference was seen between the two groups favoring LRYGB at 12 months [MD: $-0.47 \%, 95 \%$ CI: $-0.73 \%$ to $-0.20 \%$; $\mathrm{P}<0.01$ ] Subanalysis of studies including only patients living with obesity and T2DM had considerably lower numbers but generally paralleled the results from the main cohort. This systematic review concluded that LRYGB is likely more effective in short- and mid-term glucose homeostasis compared with LVSG for T2DM management (17).

This review had certain limitations, however. Among 
them was the inclusion of a study by Lee $\mathrm{et}$ al. that compared mini-gastric bypass with LVSG instead of LRYGB with LVSG (19). At 12-month follow-up, the researchers reported a T2DM remission in 28 of 30 participants (93\%) who had mini-gastric bypass and in 14 of 30 participants $(47 \%)$ in the LVSG group $(\mathrm{P}=0.02)$. This T2DM remission rate is higher in the bypass group, and lower in the LVSG group, than in most other studies. Certainly, inclusion of this non-LRYGB study could potentially bias the results. Furthermore, the majority of patients included in the primary analysis did not have a diagnosis of T2DM at baseline. It would be interesting to see the results of a systematic review in the researchers included all studies and excluded patients without T2DM at baseline.

Three other systematic reviews that reported on T2DM remission rates between LVSG and LRYGB were published between 2013 and 2015, including both RCTs and observational studies $(15,16,18)$. A study by Li et al. reported a statistically significant improved T2DM remission rate with LRYGB (OR: 1.49, 95\% CI: 1.04-2.12) (15), whereas the other 2 studies reported similar effects on T2DM remission between operations. A systematic review by Cho et al. included 857 patients, all of whom had T2DM (16). At 1 year, the T2DM remission rate between LVSG (63\%) and LRYGB (74\%) was not statistically different (RR: 0.90, 95\% CI: 0.81-1.01; $\mathrm{P}=0.07$ ), although there is a trend towards higher resolution with LRYGB. A review by Yip et al. revealed similar results, with remission rates of $76 \%$ and $68 \%$ at 1 year for LVSG and LRYGB, respectively (18).

\section{Long-term follow-up}

Although short-term remission rates are important, the long-term control of T2DM as a chronic disease is of still greater clinical significance. Currently, 3 large RCT's with 5-year follow-up provide useful information in this area: the SM-BOSS trial, the SLEEVEPASS trial, and the STAMPEDE trial $(13,20,21)$. SLEEVPASS and SMBOSS are large multicenter RCTs that primarily investigate the effect of LVSG vs. LRYGB on weight loss but include T2DM remission as secondary endpoints. Stampede, by contrast, compares intensive medical therapy $v s$. bariatric surgery (LVSG or LRYGB). Two other large trials are evaluating surgery on patients living with obesity and T2DM, reporting 5-year follow-up. However, because one compares LRYGB with BPD (22) and the other compares LVSG with mini-gastric bypass (23), they are not further discussed here.

Salminen et al. recently published 5 -year results from the Finish SLEEVEPASS trial comparing the effect of LVSG $v s$. LRYGB on excess weight loss (21). An estimated mean percentage excess weight loss of $49 \%$ was seen at 5 years after LVSG, vs. 57\% after LRYGB. At 5-year follow-up, diabetic outcomes were reported for 81 of 240 participants, producing very similar results for LVSG and LRYGB in all reported measures. Complete or partial DM remission was seen in 15 of 41 participants (37\%) after LVSG and in 18 of 40 participants $(45 \%)$ after LRYGB $(\mathrm{P}>0.99)$. Reduction in medication occurred in 21 of 41 participants (51\%) after LVSG and in 20 of 40 participants after LRYGB (50\%). HbA1c levels were also similar between groups, with mean values for both groups of $6.6 \%$. The only outcome that trended to favor LRYGB, although not to a statistically significant degree, was mean fasting plasma glucose level: $135.1 \mathrm{mg} / \mathrm{dL}$ in the LVSG group compared with $120.7 \mathrm{mg} / \mathrm{dL}$ in the LRYGB group $(\mathrm{P}=0.052)$.

Peterli et al. also recently published 5-year results of the Switzerland SM-BOSS trial comparing the effect of LVSG $v s$. LRYGB on excess weight loss (20). They demonstrated estimated mean percentage weight losses of $61 \%$ in the LVSG group and $68 \%$ in the LRYGB group. Compared with SLEEVEPASS, there were fewer individuals who had T2DM, 58 of 217 patients. However, diabetes-related outcomes were similar between surgical groups, which parallels the results of SLEEVPASS. Complete T2DM remission was seen at 5 years in 16 of 26 participants (62\%) after LVSG and in 19 of 28 participants (68\%) after LRYGB. Fasting glucose and HbA1c levels were also improved, with no difference between surgical groups. Interestingly, despite having very similar trial protocols, both mean excess weight loss and T2DM remission rates in this trial were higher compared with SLEEVEPASS.

Schauer et al. have also published the 5-year results from the STAMPEDE trial, a single-surgeon, single-institution study from the Cleveland Clinic (13). This trial, however, compares intensive medical therapy with bariatric surgery (LVSG or LRYGB) in moderately obese individuals (BMI: 27 to 43) who have T2DM and differs from previous trials in not having been designed to directly compare LVSG with LRYGB. The primary outcome of this trial was $\mathrm{HbA1c} \leq 6.0 \%$. At 5 years, 134 individuals were followed up, and the primary end point was met by 2 of 38 patients $(5 \%)$ who received intensive medical therapy alone, 14 of 49 patients $(29 \%)$ who underwent LRYGB $(\mathrm{P}=0.03)$, and 
11 of 47 patients (23\%) who underwent LVSG ( $\mathrm{P}=0.07)$. The study also reported other markers of glycemic control, including various targets of HbA1c $(\leq 6.5 \%, \leq 7.0 \%)$ and a change in fasting plasma glucose from baseline. Not surprisingly, surgical treatment was consistently superior to medical therapy alone, with no differences between LVSG and LRYGB amongst any of these endpoints. However, in terms of medication use, a significantly higher percentage of patients were not taking any glucose-lowering medications in the LRYGB group (45\%) than in the LVSG group (25\%). The results from this study suggest that LRYGB and LVSG are similar in their effectiveness in managing T2DM, although there may be a higher likelihood of being off medications with LRYGB.

\section{Conclusions}

The evidence demonstrating bariatric surgery's efficacy for improving diabetes management versus that of conventional medical care is mature and well established. In differentiating the efficacy of specific procedures, LAGB has consistently demonstrated poorer weight loss and lower T2DM remission rates, whereas BPD has consistently shown superior weight loss and higher T2DM remission rates. However, the differences in effectiveness between the two most commonly performed procedures worldwideLRYGB and LVSG-have been less clearly defined, both because LVSG is still a relatively new procedure and because the current literature is heterogeneous in methods and study design. In this review, we have sought to synthesize the best available evidence comparing LVSG with LRYGB for management of T2DM. Based on our review, we believe that both procedures are very effective at improving T2DM care, especially compared with conventional medical management. However, there may be a modest benefit to be had by using LRYGB over LVSG.

The information from this review can be of aid in selecting the ideal bariatric intervention for a particular patient. Other surgeries, including BPD, mini-gastric bypass, and single anastomosis duodenal-ileal bypass, as well as integration of novel medical treatments for weight loss and diabetes, are outside of the scope of this article. Both LVSG and LRYGB can be good options for improving T2DM care in obese patients. But patients who are strongly prioritizing optimizing T2DM management in deciding between these two surgeries may be counselled that there might be a slight benefit of LRYGB over LVSG.

\section{Acknowledgments}

Funding: None.

\section{Footnote}

Provenance and Peer Review: This article was commissioned by the Guest Editor (Muhammed Ashraf Memon) for the focused issue "Bariatric Surgery" published in Annals of Translational Medicine. The article was sent for external peer review organized by the Guest Editor and the editorial office.

Conflicts of Interest: The focused issue "Bariatric Surgery" was commissioned by the editorial office without any funding or sponsorship. The authors have no conflicts of interest to declare.

Ethical Statement: The authors are accountable for all aspects of the work in ensuring that questions related to the accuracy or integrity of any part of the work are appropriately investigated and resolved.

Open Access Statement: This is an Open Access article distributed in accordance with the Creative Commons Attribution-NonCommercial-NoDerivs 4.0 International License (CC BY-NC-ND 4.0), which permits the noncommercial replication and distribution of the article with the strict proviso that no changes or edits are made and the original work is properly cited (including links to both the formal publication through the relevant DOI and the license). See: https://creativecommons.org/licenses/by-nc-nd/4.0/.

\section{References}

1. Danaei G, Finucane MM, Lu Y, et al. National, regional, and global trends in fasting plasma glucose and diabetes prevalence since 1980: systematic analysis of health examination surveys and epidemiological studies with 370 country-years and 2.7 million participants. Lancet 2011;378:31-40.

2. GBD 2017 Risk Factor Collaborators. Global, regional, and national comparative risk assessment of 84 behavioural, environmental and occupational, and metabolic risks or clusters of risks for 195 countries and territories, 19902017: a systematic analysis for the Global Burden of Disease Study 2017. Lancet 2018;392:1923-94. 
3. NCD Risk Factor Collaboration (NCD-RisC). Trends in adult body-mass index in 200 countries from 1975 to 2014: a pooled analysis of 1698 population-based measurement studies with 19.2 million participants. Lancet 2016;387:1377-96.

4. Buchwald H, Estok R, Fahrbach K, et al. Weight and type 2 diabetes after bariatric surgery: systematic review and meta-analysis. Am J Med 2009;122:248-56.e5.

5. Gloy VL, Briel M, Bhatt DL, et al. Bariatric surgery versus non-surgical treatment for obesity: a systematic review and meta-analysis of randomised controlled trials. BMJ 2013;347:f5934.

6. Ribaric G, Buchwald JN, McGlennon TW. Diabetes and weight in comparative studies of bariatric surgery vs conventional medical therapy: a systematic review and meta-analysis. Obes Surg 2014;24:437-55.

7. Sheng B, Truong K, Spitler H, et al. The long-term effects of bariatric surgery on type 2 diabetes remission, microvascular and macrovascular complications, and mortality: a systematic review and meta-analysis. Obes Surg 2017;27:2724-32.

8. Buchwald H, Oien DM. Metabolic/bariatric surgery worldwide 2011. Obes Surg 2013;23:427-36.

9. Angrisani L, Santonicola A, Iovino P, et al. Bariatric surgery worldwide 2013. Obes Surg 2015;25:1822-32.

10. Gill RS, Birch DW, Shi X, et al. Sleeve gastrectomy and type 2 diabetes mellitus: a systematic review. Surg Obes Relat Dis 2010;6:707-13.

11. Sjöström L. Review of the key results from the Swedish Obese Subjects (SOS) trial - a prospective controlled intervention study of bariatric surgery. J Intern Med 2013;273:219-34.

12. Carlsson LM, Peltonen M, Ahlin S, et al. Bariatric surgery and prevention of type 2 diabetes in Swedish obese subjects. N Engl J Med 2012;367:695-704.

13. Schauer PR, Bhatt DL, Kirwan JP, et al. Bariatric surgery versus intensive medical therapy for diabetes - 5-year outcomes. N Engl J Med 2017;376:641-51.

14. Li JF, Lai DD, Ni B, et al. Comparison of laparoscopic Roux-en-Y gastric bypass with laparoscopic sleeve gastrectomy for morbid obesity or type 2 diabetes mellitus: a meta-analysis of randomized controlled trials. Can J Surg 2013;56:E158-64.

15. Li JF, Lai DD, Lin ZH, et al. Comparison of the longterm results of Roux-en-Y gastric bypass and sleeve gastrectomy for morbid obesity: a systematic review and meta-analysis of randomized and nonrandomized trials. Surg Laparosc Endosc Percutan Tech 2014;24:1-11.

16. Cho JM, Kim HJ, Lo Menzo E, et al. Effect of sleeve gastrectomy on type 2 diabetes as an alternative treatment modality to Roux-en-Y gastric bypass: systemic review and meta-analysis. Surg Obes Relat Dis 2015;11:1273-80.

17. Hayoz C, Hermann T, Raptis DA, et al. Comparison of metabolic outcomes in patients undergoing laparoscopic roux-en-Y gastric bypass versus sleeve gastrectomy a systematic review and meta-analysis of randomised controlled trials. Swiss Med Wkly 2018;148:w14633.

18. Yip S, Plank LD, Murphy R. Gastric bypass and sleeve gastrectomy for type 2 diabetes: a systematic review and meta-analysis of outcomes. Obes Surg 2013;23:1994-2003.

19. Lee WJ, Chong K, Ser KH, et al. Gastric bypass vs sleeve gastrectomy for type 2 diabetes mellitus: a randomized controlled trial. Arch Surg 2011;146:143-8.

20. Peterli R, Wölnerhanssen BK, Peters T, et al. Effect of laparoscopic sleeve gastrectomy vs laparoscopic Rouxen-Y gastric bypass on weight loss in patients with morbid obesity: the SM-BOSS randomized clinical trial. JAMA 2018;319:255-65.

21. Salminen P, Helmiö M, Ovaska J, et al. Effect of laparoscopic sleeve gastrectomy vs laparoscopic Roux-en-Y gastric bypass on weight loss at 5 years among patients with morbid obesity: the SLEEVEPASS randomized clinical trial. JAMA 2018;319:241-54.

22. Mingrone G, Panunzi S, De Gaetano A, et al. Bariatricmetabolic surgery versus conventional medical treatment in obese patients with type 2 diabetes: 5 year follow-up of an open-label, single-centre, randomised controlled trial. Lancet 2015;386:964-73.

23. Lee WJ, Chong K, Lin YH, et al. Laparoscopic sleeve gastrectomy versus single anastomosis (mini-) gastric bypass for the treatment of type 2 diabetes mellitus: 5-year results of a randomized trial and study of incretin effect. Obes Surg 2014;24:1552-62.

Cite this article as: Gu J, Vergis A. Diabetes improvement and bariatric surgery-review of laparoscopic Roux-en-Y gastric bypass $v s$. laparoscopic vertical sleeve gastrectomy. Ann Transl Med 2020;8(Suppl 1):S10. doi: 10.21037/atm.2020.01.47 